\title{
DOI: https://doi.org/10.24297/jap.v16i1.8525
}

\section{The Mathematical Constant of Bound Space at Value 8 And -1 Constant (Including Validation of The Created Transcendental $\mathbf{P i}, \mathbf{n}$ )}

\author{
Vinoo Cameron \\ Hope Clinic, Athens, Wisconsin \\ hope9900@frontier.com
}

\begin{abstract}
:
This paper clearly establishes by precise mathematics that the bound space of a square and the bound space of a circle are a created continuum of each other, with a validated transcendental pi as a constant between them. This validation of the transcendental $\mathrm{Pi}$ is proved at the value $8(-1)$ constant, as discovered by this author and fully expressed in this paper. The latter statement is the reality of all space and its contents of mass and speed. This paper validates by perfect mathematics / geometry the rationale behind the author's papers recently published on the inverse placement of numbers at the inverse 1:3 cone which is further explained herein at the $8(-1)$ constant.This explanatory paper is thus complementary to the published papers of the author over ten years delineating the perfect inverse placement of prime numbers at the cone of Pythagoras 1:3. Basically at the perimeter of value 8 , the 4 sided square (2:2) and a 4 sided rectangle (1:3) represent the maximal and minimal area by space at a differential of $-1\left(2^{\star} 2-3^{\star} 1\right)$ The curved bound space of a circle represents the maximal bound space at any given linear perimeter, and the paper delineates by precise equations the constants of space based on these basic observation, including the validation of the transcendental Pi by precise display of digits at a circumference of the constant value 8 .
\end{abstract}

Keywords: Bound Space, 1:3 Cone, 8 Constant Value, Transcendental Pi Value

\section{Method}

The method is based on basic geometry at the constant perimeter of value 8 . The perimeter/circumference at value 8 has a specific mathematical relationship as follows

1. At value 8 for total perimeter there is a precise -1 difference by the area between $2 \star 2$ (square) and $3^{\star} 1$ (rectangle).

2. At value 8 for total perimeter, the precise mathematical area of a circle in "square units" is equal to the value of the radius in linear units $\left(\pi r^{\wedge} 2=4 r\right)$

3. 3. The diagonal of a square with a perimeter of 8 constant is $\sqrt{ } 8$

4. By simple transposition, the square forms a pyramid with the same area, and the rectangle 1:3 forms a 1:6 cone, as is shown in the diagram. 


\section{Diagram:}
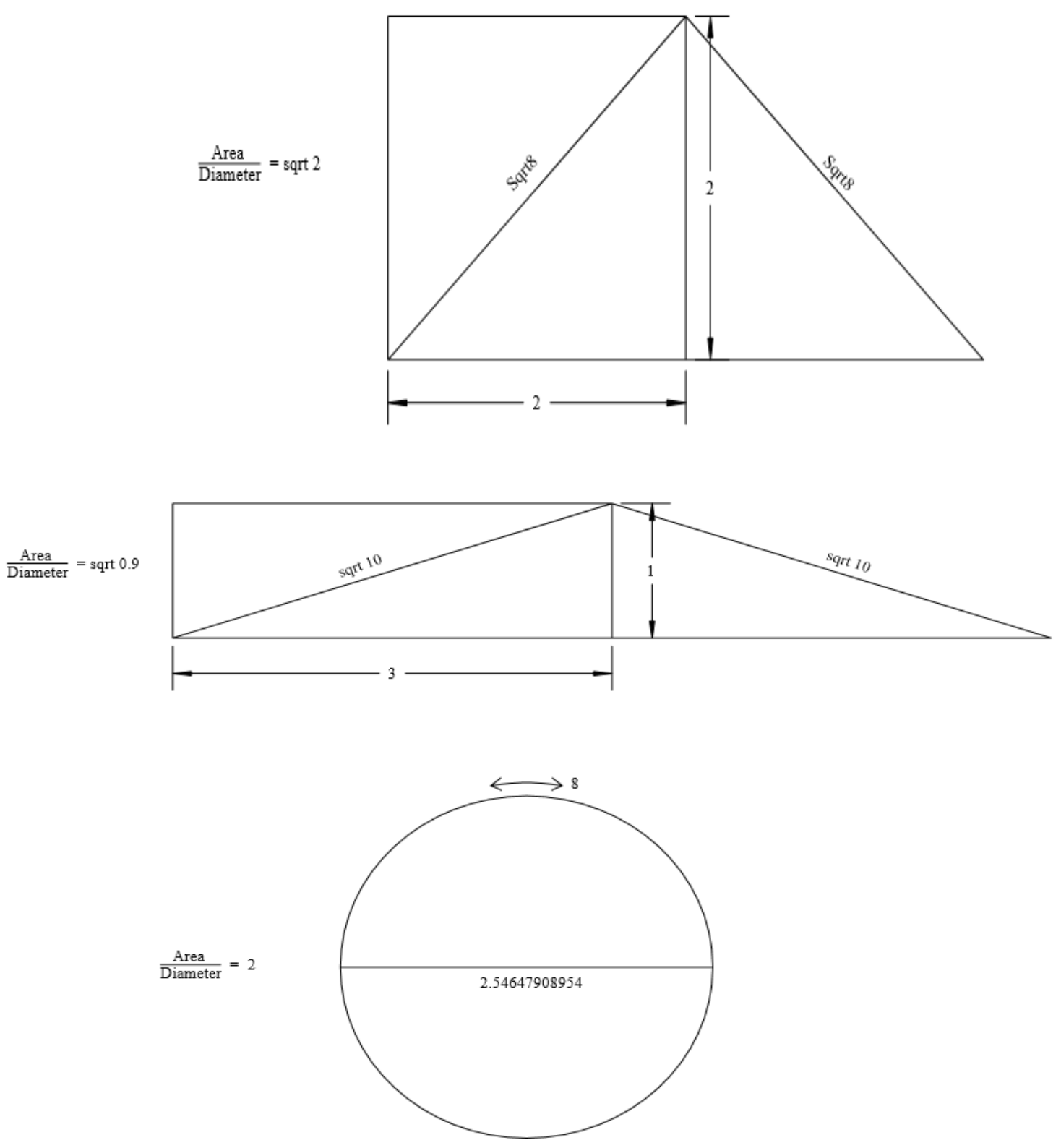

The whole number 1 value, as created, is the base number both by geometry and numbers. As clearly demonstrated in published papers referenced here, Prime numbers and their derivatives are spirally placed at inverse 1:3, which is represented by the rectangle at 1:3 in the diagram. From that base of number 1 , numbers are arranged in order, and that order extends to both the linear and the non- linear geometric configuration as a continuum. The author has chosen the value 8 for three specific reasons.

1. At value 8 exclusively, there is a precise -1 difference by area between the same perimeter of 8 between $2 * 2$ (square) and $3 * 1$ (rectangle).

2. At value 8 mathematically the area of a circle in square units is equal to the value of the radius in linear units $\left(\pi r^{\wedge} 2=4 r\right)$

3. 3. The diagonal of a square with a perimeter of 8 constant is $\sqrt{ } 8$ 


\title{
The Validation of the Transcendental $\mathrm{Pi}$ as a created value by $8(-1)$ Constant
}

\begin{abstract}
The cardinal equation at $\mathrm{Pi}$ value 3.14159265358 is unique to this $\mathrm{Pi}$ value by precise digits at 8 constant circumferences and a square with 8 perimeter and diagonal at $\sqrt{ } 8$ (2.82842712475) computed with the diameter of the circle and area of the circle at circumference 8 . Note the unique digits and the precise division which is unique to this $\mathrm{Pi}$ value
\end{abstract}

$3.14159265358 * 2=6.28318530716$

$8 / 6.28318530716=1.27323954474$ (Radius)

$1.2732354474 * 2=2.54647908948$ (Diameter).

$3.1459265358 *(1.27323954474) \wedge 2=5.09295817896$ (area of a circle)

$5.09295817896 / 2.82842712475=1.800,632,632,16$

$2.5464780948 / 2.82842712475=0.900,316,316,32$

$1.800,632,632,16 / 0.900,316,316,16=2$ precise

Here are the precise cardinal values at 18 digits of the Pi, as derived by the above calculus.

\section{Transcendental Pi value at 3.14159265358977323846264. at constant 8(-1)}

At precise 18 by the same basic equation as above, the value is also 2 precise as follows in thee precise digits order, in fact precisely at the Transcendental Pi value at 18 digits all the "3-digit sets" divide precisely perfectly as 2. Please note this carefully, as shown. The author has not researched this beyond 18 digits computation; it is not necessary

$$
\frac{1.800,632,632,314,212,138}{0.900,316,316,157,106,069}=2 \text { precise }
$$

There are many in the world of mathematics such as the Cosmic Pi, and the Golden Ratio, but here is the example of one Cosmic Pi of a Sarva Reddy or Harry Lear, it does not fit the mold by far.One such Pi value touted in the world is $\mathrm{Pi}=3.14644660941$; there is no need to prove all these other values that have challenged the Transcendental Pi value in the world today. Here is the comparison of one such Pi value by the same quadratic equation, there are no such phenomena with this Pi value or any other. The transcendental Pi is the created correct value.

\section{Example of Incorrect Pi Value (Cosmic Pi, Golden ratio, etc.)}

$$
\frac{1.797,569,184,22}{0.898,784,592,11}=2 \text { precise }
$$

There is no comparative fractionization by digits here, so this Pi and all others are incorrect.

\section{Explanatory notes.}

$\checkmark(8)$ in diameter at the square at 8 constant perimeter $=2.828427124752$

At the specific and exclusive Pi value $3.1415926535 . . .$, a circle at the circumference of 8 , by simple calculation, the precise value of the radius $=1.27323954477$, the precise diameter $=2.54647908948$. The precise area at circumference of $8\left(\pi r^{\wedge} 2\right)=5.09295817908$. Thus, 
1. The area of a circle at the circumference of 8 divided by the area of a square at perimeter of 8 is equal to the radius of the circle at circumference of 8 as,

2. $5.09295817908 / 4=1.27323954477$ radius $\left(\pi r^{\wedge} 2=4 r\right)$

3. 2. The area of a circle at circumference of 8 divided by diagonal of square at perimeter 8 is

$5.09295817908 / \vee 8=1.800,632,632 \ldots$

3.

Diameter at the circle at 8 circumferences, 2.54647908948

Diameter at square with 8 perimeter, 2.82842712475

$\frac{2.54647908948}{2.82842712475}=0.900,316,316 \ldots$

Furthermore, from above :1.800,632,632/0.900,316, 316=2. These derived values at the Pi value 3.415926535 are unique, and the digital phenomena of "repeat digits" shown here are exclusive to the base Pi value 3.1415926535 and suggest the parity of the square and circle at 8 constant $(-1)$ perimeter. The validation is solid in that the transcendental PI number digits 3.1415926535 exclusively tie/ arrange the square at 8 perimeters and the circle at 8 circumference as a mathematical continuum of bound space by "specific digits arrangement" that no other PI value can generate. Note digits $(90),(45)$ and the precise divisions as shown under,

There is also this Plethora of repeat numbers at the circle generated at the Pi digits 3.1415926535 at 8 Circumference;

$0.900316,316 / 2=0.450,158,158$

$0.900316,316 / 8=0.1125,395,395$

$0.1125395,395, / 2=0.05626,697,697$

$0.450,158,158 / 2=0.2250,790,790$

$0.450158,158 / 8=0.00562,697,697$

$$
\sqrt{\frac{2.54647908954}{3.1415926535}}=\left(\frac{2.54647908954}{2.82842712475}\right)=0.900316316 \ldots
$$

$\sqrt{ }($ Diameter of circle $/ \mathrm{Pi})=($ Diameter of circle $/$ diameter at square $)=0.900316316$

Thus, in synopsis, the transcendental Pi base digits are precise: It is a clear, direct continuum relationship between a linear square at 8 perimeter and a curved circle at 8 circumferences, tied/arranged by the Pi value digits which are fundamental transcendental at $3.1415926535 \ldots$ as starter non- algebraic digits of the Pi value. Moreover, that relationship is clearly by very persistent repeating numbers between the two values (square/Circle at 8), as is shown above. Apparently, the irrational digits of the Pi exclusive to these equations (3.415926535), generate specific "rational repeat of digits" as shown above and concluded below. By values specifically derived by the PI 3.1415926535

Area of a square and circle at 8 / Diameter of the square and circle respectfully at 8 are as follows

At Circle: $5.09295817908 / 2.54647908954=2$ 
At Square: $4 / \sqrt{ } 8=\sqrt{ } 2$

At rectangle $1: 33 / \sqrt{ } 10=\sqrt{ } 0.9$

$5.09295817908 / \sqrt{ } 8=1.800,632,632$ (unique to 3.1415926535 )

Conclusion and discussion: In the understanding of this paper and the published papers on the spiral placement of numbers, the reason that the prime numbers are spirally placed at the half-line of the $1: 3$ as shown in my published papers is that prime numbers are created at and for the -1 space of 1:3 rectangle, with the 1:3 cone and the 1:6 curve described in the diagram in this paper and the 1:6 Curve described in the other papers. Any two points in the universe are inherently curvi- linear to each other as in the 1:6 curve. The 1:6 constant curve has its basis in the rectangle 1:3 above and has been discussed in the previous physics paper and since this is the only exclusive curve whole number curve at 1 vertical in the whole of geometry at 1 vertical and 6 bases by Pythagoras theorem, this diagram is inserted below as reference.

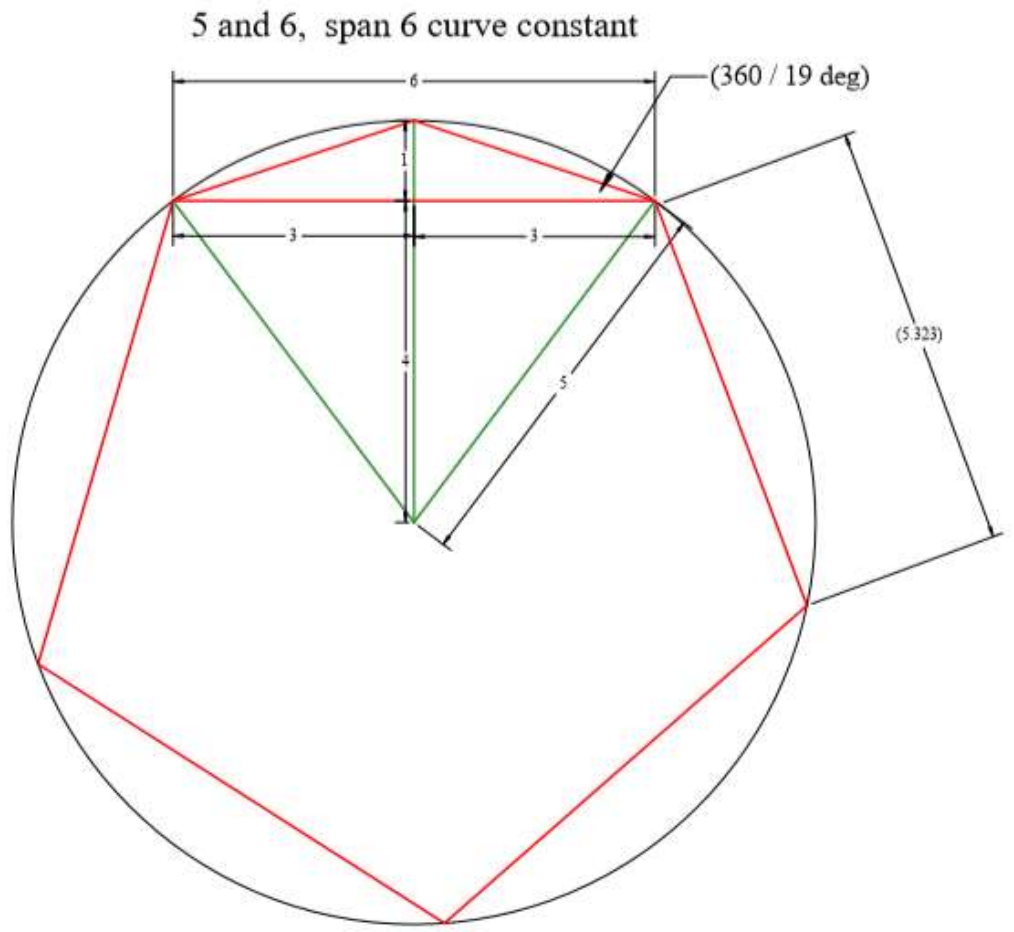

The above resolution is the basis for all finite bound space in the universe, expressed by precise numbers and constants. The papers published by Math-lab and JAP, are a clear representation of these constants at the finite inverse cone 1:3. The "quanta of space, mass, and energy" is, as a rule are bound by numbers constants, as is the "Max Planck's" constant at $6+$. As shown at 8 constant $(-1)$, by specific PI value of $3.1415926535 \ldots . .$. , the Transcendental $\mathrm{Pi}$ is created as a continuum to the square and the circle as evident at the constant $8(-1)$ by set numbers as shown precisely at this paper and the recent published papers on the inverse spiral arrangement of finite prime numbers at 1:3 cone and rectangle.

\section{References:}

1. Cameron, V. (2018). Math-LAB Journal, 1(3), 343-345. "The Parody of Mathematics"

2. Journal of Advances in Physics, Volume16(2019) ISSN 2347-3487: "The infinite inverse placement of prime numbers and their derivatives at the cone of Pythagoras1:3".

3. Journal of Advances in Physics, Volume 16 (2019) ISSN: 2347-3487 https://cirworld.com/index.php/jap: "The Inverse precise placement of numbers based at Pythagoras 
1:3 and the precise configuration of numbers as the basis of a unified theorem that binds the physics of mass, space, energy and the torque power(inertia) that sustains the finite universe. (Section 11, Physics).

4. Journal IJAMR. Paper on the pure mathematics of the inverse spiral arrangements of prime numbers, due to be published soon. 